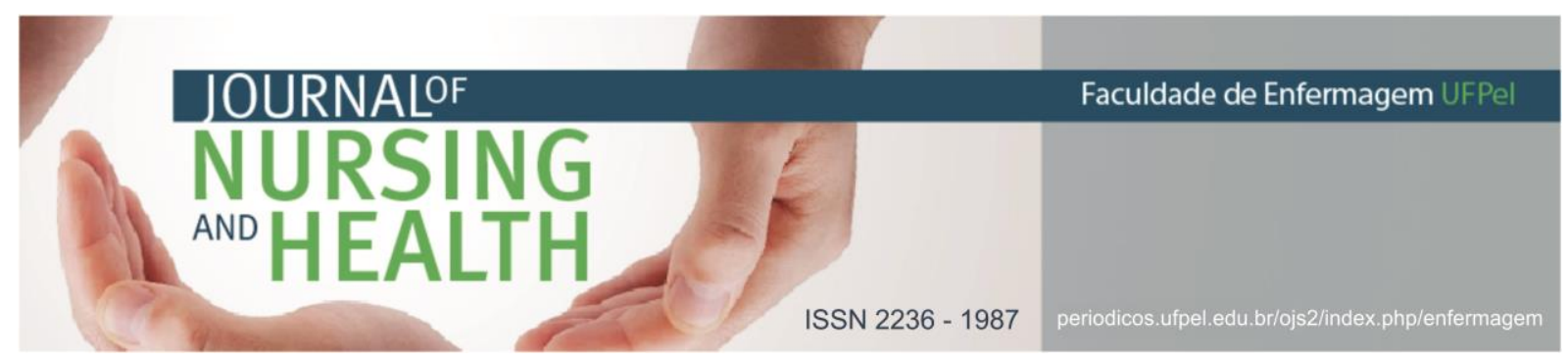

ARTIGO ORIGINAL

\title{
O cuidado a hipertensos e diabéticos na perspectiva da Política Nacional de Humanização
}

\section{Care for hypertensive and diabetic patients in the perspective of the National Humanization Policy}

\section{El cuidado de hipertensos y diabéticos en la perspectiva de la Política Nacional de Humanización}

Curioletti, Regina Maria ${ }^{1}$; Colliselli, Liane²; Madureira, Valéria Silvana Faganello³ Tombini, Larissa Hermes Thomas ${ }^{4}$

\begin{abstract}
RESUMO
Objetivo: conhecer como os profissionais da equipe de saúde da família conduzem o processo de cuidar de portadores de diabetes mellitus e hipertensão arterial sistêmica. Métodos: trata-se de estudo exploratório descritivo de abordagem qualitativa desenvolvido em um Município do Norte do Estado do Rio Grande do Sul no segundo semestre de 2014, com um grupo de dez portadores de diabetes e hipertensão e três profissionais da equipe de saúde da família. A análise temática foi empregada para o tratamento dos dados e tomando a Política Nacional de Humanização como referencial teórico. Resultados: demonstram avanços em relação a implementação da Política Nacional de Humanização e desafios em relação ao projeto terapêutico singular e à clínica ampliada. Considerações finais: conclui-se que ações de educação permanente, na perspectiva da Política Nacional de Humanização são necessárias visando a qualificar o cuidado.

Descritores: Atenção primária à saúde; Doença crônica; Humanização da assistência; Educação em enfermagem.
\end{abstract}

\section{ABSTRACT}

Objective: to know how the professionals of the family health team lead the process of caring for patients with diabetes mellitus and Systemic Arterial Hypertension. Methods: this is a descriptive exploratory study of a qualitative approach developed in a municipality in the North of the State of Rio Grande do Sul in the second half of 2014, with a group of ten patients with diabetes and hypertension and three professionals from the family health team. The thematic analysis was used to treat the data taking the National Humanization Policy as a theoretical reference. Results: it demonstrates advances in relation to the implementation of National Humanization Policy and challenges with singular therapeutic project and the extended clinic. Final considerations: it is concluded that permanent education actions, in the perspective of the National Humanization Policy, are necessary in order to qualify the care.

Descriptors: Primary health care; Chronic disease; Humanization of assistance; Education, nursing.

\footnotetext{
1 Enfermeira. Graduada pela Universidade Federal da Fronteira Sul (UFFS) Chapecó/SC. E-mail: valeria.madureira@uffs.edu.br http://orcid.org/0000-0002-9197-6492

2 Enfermeira. Mestre em Enfermagem. Universidade Federal da Fronteira Sul (UFFS) Chapecó/SC. E-mail: liane.colliselli@uffs.edu.br http://orcid.org/0000-0002-2774-5083

3 Enfermeira. Doutora em Enfermagem. Universidade Federal da Fronteira Sul (UFFS) Chapecó/SC. E-mail: valeriamadureira2005@hotmail.com http://orcid.org/0000-0001-7990-3613

${ }^{4}$ Enfermeira. Mestre em Enfermagem. Universidade Federal da Fronteira Sul (UFFS) Chapecó/SC. E-mail: larissa.tombini@uffs.edu.br http://orcid.org/0000-0002-6699-4955
} 


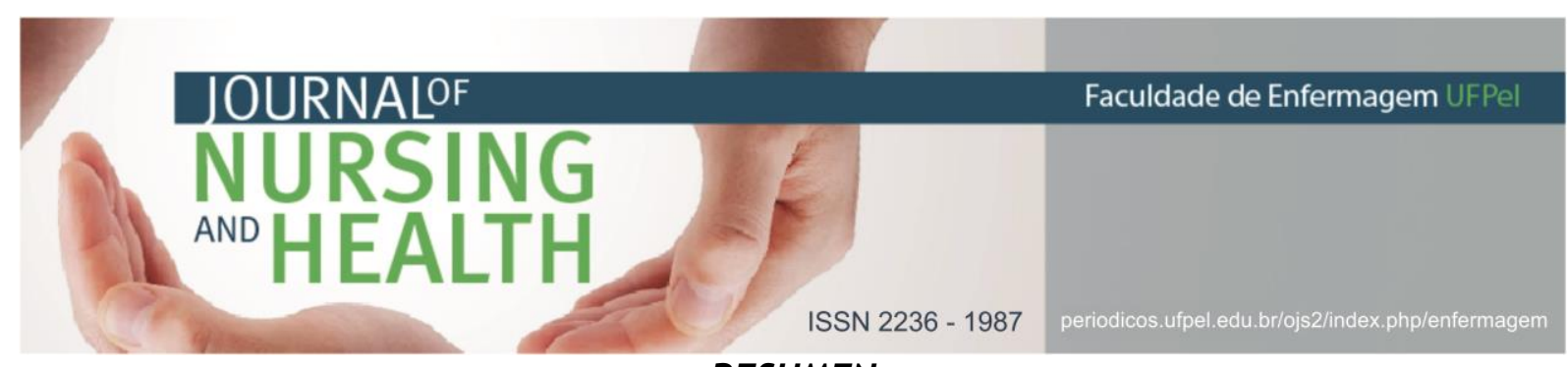

RESUMEN

Objetivo: conocer cómo los profesionales del equipo de salud de la familia conducen el proceso de cuidar de portadores de diabetes mellitus y Hipertensión Arterial Sistémica. Métodos: se trata de un estudio exploratorio descriptivo de abordaje cualitativo desarrollado en un Municipio del Norte del Estado de Rio Grande do Sul en el segundo semestre de 2014, con un grupo de diez portadores de diabetes y hipertensión y tres profesionales del equipo de salud de la familia. El análisis temático fue empleado para el análisis de los datos tomando la Política Nacional de Humanización como referencial teórico. Resultados: demuestran avances en relación a la implementación de la Política Nacional de Humanización y desafíos en relación al proyecto terapéutico singular y a la clínica ampliada. Consideraciones finales: se concluye que acciones de educación permanente, en la perspectiva de la Política Nacional de Humanización, son necesarias para calificar el cuidado.

Descriptores: Atención primaria de salud; Enfermedad crónica; Humanización de la atención; Educación en enfermería.

\section{INTRODUÇÃO}

As Doenças Crônicas Não Transmissíveis (DCNT) têm gerado elevado número de mortes prematuras e são de etiologia multifatorial. ${ }^{1}$ São responsáveis por $75 \%$ das mortes no Brasil. $^{2}$ Os hábitos modificáveis das DCNT como os alimentares inadequados, 0 sedentarismo, 0 tabagismo são relevantes para adoção de estilo de vida saudável, o que envolve questões comportamentais que apresentam maior custoefetividade. $^{3}$

Entretanto, os usuários apresentam dificuldades com essas restrições em seus hábitos, tendo sentimento de impotência ao não verem de imediato os resultados após a adoção de um estilo de vida saudável. ${ }^{4}$ Atenta a isso, a Política Nacional de Humanização (PNH) traduz os princípios do Sistema Único de Saúde (SUS) para os diferentes sujeitos do sistema, realizando trocas solidárias entre sistema e usuários, bem como disponibilizando ações humanizadoras. A política se dá pela troca de conhecimento, observação das necessidades, interação harmoniosa entre esferas de governo, trabalho multidisciplinar e pelo resgate dos princípios do SUS. ${ }^{5} \mathrm{~A}$ forma como o usuário é acolhido no sistema pode gerar boa interação ou conflitos e a falta de acolhimento humanizado poderá interferir no processo de cuidado.

0 presente estudo, focado no cuidado a portadores de Hipertensão Arterial Sistêmica (HAS) e Diabetes Mellitus (DM), se fundamenta na situação das DCNT no mundo e no município onde foi desenvolvido, no qual cerca de $15 \%$ da população é portadora de HAS e aproximadamente 2,8\% de DM. ${ }^{6}$ Teve como objetivo conhecer como os profissionais da Equipe Saúde da Família (ESF) conduzem o processo de cuidar de hipertensos e diabéticos.

\section{MATERIAIS E MÉTODOS}

Trata-se de pesquisa com abordagem qualitativa, de caráter exploratório descritivo, desenvolvida no segundo semestre de 2014 em um Município do Norte do Rio Grande do Sul com cerca de quatro mil habitantes. O município tem 1.266 


\section{JOURNALF \\ NURSING \\ ANO HEALTH}

ISSN 2236 - 198

à gestão de trabalho, participação em capacitações, facilidades e dificuldades encontradas no cuidado aos pacientes HAS/DM e aspectos relacionados ao cuidado conduzido pelos profissionais contemplando a Política Nacional da Humanização.

Para o tratamento dos dados utilizou-se a técnica de análise temática de Minayo ${ }^{7}$ e emergiram as seguintes categorias: 'acesso ao serviço', 'acesso à informação', 'cuidado' e 'participação'. Os sujeitos do estudo foram identificados por letras seguidas de números, $P$ (profissional) e U (usuário). 0 projeto foi aprovado pelo Comitê de Ética em Pesquisa da Universidade Federal da Fronteira Sul (UFFS) (parecer $\mathrm{n}^{\circ}$ 750.639).

\section{RESULTADOS E DISCUSSÃO}

A análise inicial dos prontuários eletrônicos indicou ausência de registros dos atendimentos feitos aos usuários, dentre os quais a Consulta de Enfermagem (CE). Esse fator limitou a obtenção de dados relacionados à trajetória do cuidado especialmente relativos ao Hemoglicoteste (HGT), resultados de exames, anamnese, exames físicos, registro de visitas domiciliares, diagnósticos de enfermagem e planos de cuidados.

A CE é a metodologia científica de trabalho do enfermeiro que "confere maior segurança aos pacientes, melhora a qualidade da assistência e concede maior autonomia aos profissionais de enfermagem". 8:9 Prevista pela Resolução COFEN $\mathrm{n}^{\circ} 358 / 2009$, a CE é atividade obrigatória do enfermeiro desenvolvida deliberada e 


\section{A}

sistematicamente em todos os ambientes públicos e privados em que ocorre 0 cuidado profissional de enfermagem. ${ }^{9}$ Tal como qualquer outra atividade envolvida no cuidado, a CE deve ser formalmente registrada no prontuário.

Nos usuários participantes, oito mulheres e dois homens, a idade variou entre 53 e 88 anos. No grau de escolaridade prevaleceu 0 ensino fundamental incompleto. A maioria residia na área rural do município e eram aposentados da agricultura. Os profissionais participantes eram do sexo masculino, com idade ente $31 \mathrm{e}$ 48 anos e titulação de especialistas, dois em saúde da família.

Muitos usuários tiveram dificuldade em situar o início da HAS/DM no tempo, mas informaram ser portadores há no mínimo 5 e no máximo 29 anos. Tal informação provavelmente baseou-se no início do tratamento, considerando o caráter silencioso dessas doenças. Os relatos revelam história familiar de HAS e DM:

[...] uns 25 anos, daí para mais! Descobri por que fui consultar $e$ fiz exames. [...] a pressão estava lá em cima sempre e a diabetes também [...] (U7).

[...] não sei! Minha avó tinha diabetes, mas não se tratava [...] (U4).

Os dados demonstraram fatores relacionados aos hábitos de vida, como em um estudo realizado sobre doenças crônicas não transmissíveis em adultos residentes em capitais do Brasil. ${ }^{10}$ Evidenciam também longo convívio com a doença, seu caráter silencioso,
ISSN $2236-198$

bem como complicações crônicas decorrentes da condição de saúde/doença.

Percebeu-se comprometimento oftalmológico, neuropatias, pé diabético e problemas cardiovasculares, o que requer monitoramento periódico pela equipe de saúde na perspectiva do cuidado integral, com o objetivo de evitar e ou minimizar a agudização de complicações crônicas. A seguir serão discutidas categorias e subcategorias identificadas na análise.

\section{Acesso ao serviço}

0 acesso aqui abordado se refere ao caminho que o usuário dispõe para utilizar os serviços da UBS, caracterizado pelo acolhimento e, sendo compreendido enquanto dispositivo capaz de reorganizar a atenção à saúde para atender as demandas da população, com práticas em saúde humanizadas, bem como de possibilitar o vínculo entre usuários e profissionais, estimulando ao autocuidado e corresponsabilização na terapêutica proposta. ${ }^{11}$

Destacaram-se manifestações positivas tanto por parte do usuário, como dos profissionais, mas também se evidenciou a dificuldade de acesso para consulta médica:

[...] nunca fiquei sem ser atendida, nunca faltou remédio [...] (U4).

[...] três vezes que vou para mostrar exame e não acho médico [...] (U8). 
[...] 0 acolhimento é principalmente realizado pelo enfermeiro na unidade. A gente gosta de fazer uma escuta qualificada, terapêutica mesmo, para direcionar o usuário para a sua categoria dentro das suas necessidades [...] (P3).

A UBS é a principal porta de entrada do SUS, com o papel de reconhecer o conjunto de necessidades em saúde e organizar respostas adequadas e oportunas que tenham impacto positivo nas condições de saúde do indivíduo ${ }^{12}$, o que é evidenciado nas falas pela menção à escuta qualificada.

Já a falta de médico na UBS evidenciada pelo usuário 8 reflete a situação vivida por muitos pequenos municípios e por áreas mais remotas do país. Tal estado de coisas motivou o Ministério da Saúde (MS) à proposição do Programa Mais Médicos, pois a falta desse profissional acrescida "a déficits de acesso e qualidade na atenção básica são problemas que dificultam 0 avanço do SUS". ${ }^{13: 46}$

Identificou-se que os participantes, portadores de DCNT, não têm acompanhamento periódico programado, conforme preconiza o MS:

[...] se você quer ser atendido antes, você vai mais cedo $e$ senão, vai mais tarde e tem também [...] (U2).

[...] você vai lá e eles já te atendem $[\ldots]$ (U3).

[...] Toda vez que chega, é atendido pelo médico ou pelo enfermeiro (P2).
[...] várias ações da UBS são por demanda espontânea; algumas são programadas: dentistas, nutrição, fonoaudiologia, parapsicologia, fisioterapia, as práticas integrativas [...] (P3).

A PNH preconiza a diminuição das filas, do tempo de espera e ampliação do acesso, bem como acolhimento baseado em critérios de risco com vistas à resolutividade. ${ }^{14} \mathrm{~A}$ organização da demanda para portadores de DCNT com agendamento e programação de consultas contribuiria para melhorar o acesso e a qualidade dos serviços.

\section{Acesso à informação}

0 acesso à informação implica em estabelecer relações de comunicação, informação, tratamento e diálogo entre os trabalhadores e usuários no âmbito dos serviços de saúde. ${ }^{15}$ As informações emitidas pelos profissionais em relação ao cuidado à saúde, muitas vezes não são apreendidas ou são parcialmente compreendidas:

[...] os médicos não me agradam nada [...] aquele que tem aí não se entende nem o que ele fala [...] (U10).

[...] você esbarra no paciente que é idoso, que não consegue (entender), aí você orienta, aí tem a questão cultural, aí você pede para ele mudar o estilo de vida e ele não consegue mudar, aí você aumenta a dose do remédio e continua não funcionando. Eu acho que a saúde depende muito da questão educacional sabe? (P1). 


\section{ISSN 2236 - 1987}

Os relatos sugerem barreiras de comunicação decorrentes do baixo nível de escolaridade, resistência a mudanças de hábitos de vida e ao domínio da língua. Isso impõe um grande desafio aos profissionais da saúde, que precisam pensar alternativas para facilitar a compreensão do usuário sobre informações relativas à sua condição de saúde.

0 acesso à informação remete ao estabelecimento de vínculo e identificou-se dificuldade de compreensão por parte dos usuários, o que pode resultar em não adesão ao tratamento. A PNH preconiza troca de conhecimentos, observação das necessidades, interação harmoniosa e diálogo entre profissional e usuário. ${ }^{13}$ Compreende-se que a equipe deverá utilizar estratégias para que os usuários compreendam sua condição, reconheçam sinais e sintomas de possíveis complicações e procurem a UBS quando necessário. Assim, o processo de inclusão é uma atitude generosa capaz de produzir um processo de gerir e cuidar, considerando os princípios e diretrizes do SUS através de embasamento nos pressupostos éticos. ${ }^{14}$

\section{Cuidado}

Ao portador de doença crônica faltam cuidados planejados que possam prever suas necessidades para proporcionar atenção integrada. ${ }^{4} \mathrm{Com}$ base nessa afirmação e na $\mathrm{PNH}$ utilizou-se o conceito de cuidado de Boff, que o define como mais do que um ato; como uma atitude, o que implica mais que um momento de atenção, de zelo, de desvelo, mas representa uma atitude de ocupação, preocupação, de responsabilização e de envolvimento afetivo com o outro. ${ }^{16}$ Cuidado refere-se às relações de amor ou afeto estabelecidos entre os seres vivos. ${ }^{16}$

Aqui destacaram-se o modelo biologicista ainda utilizado pelo médico e dificuldade da equipe de enfermagem em abordar o usuário:

[...] elas atendem bem as gurias lá na farmácia, mas os médicos já despacham a gente. Não deu para falar tudo que a gente gostaria de dizer e já te mandam embora [...] é tudo rápido [...] (U8).

[...] muitas vezes não temos recurso humano para intervir naquela dificuldade [...] às vezes há desiquilíbrio dentro do próprio quadro para abordar o usuário, porque a equipe não está preparada [...] (P3).

0 atendimento apresenta fragilidades, é rápido, com pouco diálogo entre usuário e profissional, o que certamente compromete o estabelecimento de vínculo e a qualidade do cuidado. A fala do profissional permite identificar limites que a falta de integração e interação na equipe interpõe ao atendimento. Logo, em relação à CE, os participantes afirmam:

[...] nunca fiz com o enfermeiro, nem sei quem é. Sempre consultei com o doutor [...] (U2).

[...] a gente consegue acompanhar a maior parte de pacientes hipertensos $e$ diabéticos nos grupos com 


\section{ISSN $2236-1987$}

orientações, renovações das receitas. [...] com pacientes que moram muito longe, a maiorias das indicações dos medicamentos é feita nos grupos. O retorno geralmente é em seis meses. Os enfermeiros atuam mais na grupalidade (P3).

Os relatos evidenciam que 0 enfermeiro não é reconhecido pelo usuário como integrante da equipe de saúde. Este profissional remete sua atenção ao paciente HAS/DM em atividades coletivas desenvolvidas através de grupos educativos organizados. A CE não está incluída na rotina da assistência ao portador de doença crônica e, caso seja realizada, não se identificou registro no prontuário do paciente. Embora as atividades com grupos representem parte importante da atuação do enfermeiro na Atenção Básica $(A B)$, percebe-se que, nesse caso, são utilizadas principalmente para a renovação de receitas e para o repasse de informações, o que sugere uma prática de atenção à saúde prescritiva e centrada na doença.

Em relação ao acompanhamento no domicílio, foram mencionados os profissionais que atuam nas visitas, bem como a periodicidade delas mesmas e a definição de prioridades:

[...] agente de saúde (U1).

[...] faz um ano vieram a enfermeira e o médico (U4).

[...] toda a semana, dois dias para fazer visita [...] no primeiro momento estamos priorizando pacientes com hipertensão $e$ diabetes (P2).
A visita domiciliar é ferramenta para ir ao encontro daqueles impossibilitados de se deslocarem até a UBS ou distantes do serviço. 0 domicílio é um espaço singular para o cuidado, vínculo, acolhimento e humanização. ${ }^{17}$

No que se refere aos medicamentos, todos demostraram domínio e compreensão, o que é avaliado como positivo no cuidado ao portador de HAS/DM em uso de terapia contínua e para o serviço, pois sugere eficácia da educação em saúde promovida pelo serviço:

[...] eles deram para ela uma caixinha. Está separado o que tem de tomar de manhã e de noite. Aí ela tem tudo bem colocadinho [...] (U10).

[...] os pacientes chegam no posto, têm uma carteirinha onde são anotados os medicamentos, a dosagem e eles retiram a insulina (P1).

Além dos medicamentos, $80 \%$ dos participantes têm glicosímetro em casa, o que permite acompanhamento do diabetes. Porém, há fragilidade na utilização desse recurso, pois constatou-se que poucos realizam o teste diariamente, enquanto outros não sabem como usar o aparelho e nem como interpretar os resultados:

[...] às vezes faço todos os dias, às vezes faço a cada 3-4 dias. Enjoa furar o dedo todo o dia [...] ganhei no posto, [...] (U8).

[...] ah, aquele que fura o dedo? Sim tenho, mas não sou capaz de 


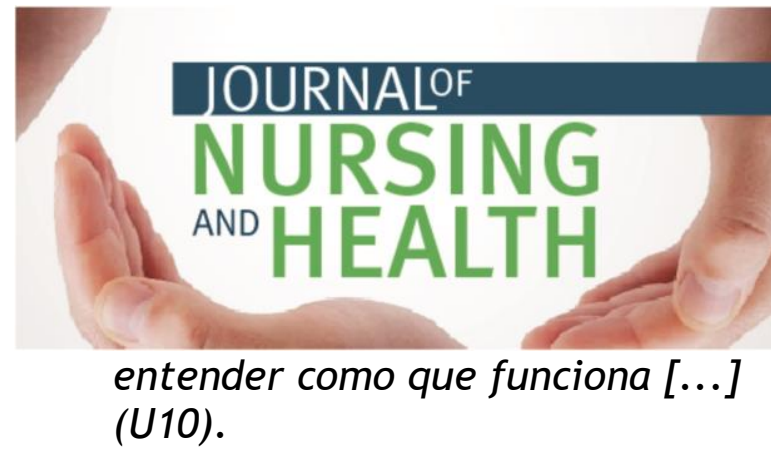 \\ [...] o correto seria a cada três meses realizar os exames de hemoglobina glicosilada, glicose de jejum e fazer um mapa glicêmico que deveria ser medido três vezes ao dia antes do café, antes do almoço e antes de ir dormir, só que na verdade poucos pacientes fazem isso ( $P 1)$.}

Percebe-se que os profissionais do serviço precisam capacitar os usuários e/ou familiares para a utilização diária correta do glicosímetro. Entretanto, é importante considerar as dificuldades que usuários muitas vezes idosos e ou com baixa escolaridade e ou dependentes de outrem para aferir a glicemia capilar têm para fazê-lo três vezes ao dia. Daí a necessidade de o cuidado ir ao encontro das condições concretas de existência dos usuários para aumentar as possibilidades de adesão ao tratamento.

0 portador de doença crônica carece de cuidados planejados, capazes de prever suas necessidades e proporcionar atenção integrada, o que demanda treinamentos para que aprenda a cuidar de si em sua residência. $E$ é tanto maior a possibilidade de que isso ocorra quanto maior for a proximidade entre 0 cuidado recomendado, a forma de executá-lo e as condições de vida e de compreensão dos usuários. Conhecimento do território, visita domiciliar e consulta de enfermagem são ferramentas essenciais para tanto.

Considerando as complicações crônicas decorrentes das doenças e a
ISSN 2236 - 1987

necessidade do acompanhamento especializado na perspectiva do cuidado integral, identificou-se que poucos usuários realizam acompanhamento com o cardiologista e endocrinologistas, entre outros. Observou-se que os que já realizaram consulta especializada pagavam parte do valor da consulta ou utilizavam planos de saúde privados.

[...] sim, com o cardiologista, mas é através do plano privado [...] (U1).

[...] sim, o posto encaminha, mas eu pago particular [...] (U3).

[...] quando necessário sim, é realizado encaminhamento [...] para o cardiologista com maior frequência ou eventualmente para o endocrinologista [...] (P1).

Os relatos demonstram uma lacuna nos serviços disponibilizados pelo SUS no que se refere a serviços de referência e contra referência. A equipe de atenção básica precisa ser resolutiva na coordenação do cuidado, tendo essa como a porta de entrada preferencial do sistema de saúde, nas diferentes fases da vida das pessoas e seus problemas de saúde, encaminhando adequadamente para outros serviços, sem deixar de ser corresponsável pela continuidade do cuidado ao longo do tempo. ${ }^{18}$ Para o sucesso das atividades da $A B$, a gestão do serviço deve estar comprometida com o trabalho em redes de referência e contra referência, que devem possuir postura ético/política comprometida com as necessidades do indivíduo. A atenção primária é um importante local para disponibilizar cuidados aos 
problemas e agravos em saúde, proporcionando atendimento longitudinal, serviços assistenciais e não assistenciais e reforçando o compromisso com as redes de atenção. ${ }^{14}$

No entanto, a disponibilidade dos serviços de referência, especialidades ou apoio matricial é uma fragilidade identificada. Não foi possível identificar, nos registros do prontuário e na fala dos usuários, como os princípios da Clínica Ampliada e do Projeto Terapêutico Singular (PTS) são colocados na prática cotidiana dos serviços. Conforme a PNH, clínica ampliada implica compromisso radical com o sujeito doente, visto de modo singular, bem como em assumir a responsabilidade sobre os usuários dos serviços de saúde, com ajuda de outros setores e "reconhecendo os limites dos conhecimentos dos profissionais e das tecnologias por eles empregadas, bem como buscar outros conhecimentos e assumir um compromisso ético profundo". 19:13

O PTS requer coprodução e cogestão do processo terapêutico de indivíduos e grupos em situações de vulnerabilidade. Já a singularidade se caracteriza pela compreensão do usuário como sujeito singular junto com o qual é determinada a ação de saúde para produzir saúde. ${ }^{19}$ Isso implica compromisso e corresponsabilização, pois o processo terapêutico é construção coletiva entre profissionais e usuário.

\section{Participação}

Esta categoria se refere à participação nas atividades de grupo e, também, à participação dos profissionais na gestão das políticas de saúde do município. Dos participantes, seis informam participar das atividades mensais do grupo de hipertensos e diabéticos. Os demais não participam por dificuldades de deslocamento até o local do encontro pela distância ou por condições de saúde que demandam auxílio de familiares. Há satisfação e descontentamento com os temas abordados no grupo:

\section{[...] sim, eu gosto, acho que vale} a pena né. A gente aqui no interior é sempre bom saber coisas diferentes [...] (U9).

[...] não dá graça, cansa de ficar lá sentado, eu não gosto (U8).

[...] eles deviam falar de outras doenças, porque toda vez é a mesma: só diabetes. Falassem de outras, tem tantas [...] (U7).

[...] a gente pauta muito no nosso serviço a prevenção e promoção da saúde, principalmente temas como nutrição, uso dos medicamentos. Partir do que o usuário tem, para fortalecer a autonomia (P3).

As atividades grupais são pontos fortes da ESF e ocorrem periodicamente em distintos espaços da comunidade, tendo como foco temáticas relacionadas às doenças e formas de promoção da saúde e de prevenção de agravos. Porém, as falas indicam também o caráter monótono e repetitivo de tais atividades, que contemplam sempre temáticas relacionadas à mesma doença, o que desmotiva a participação dos portadores de HAS/DM. Muitas vezes, 


\section{ISSN 2236 - 1987}

as atividades do grupo contemplam mais a doença do que o indivíduo, repassando uma receita pronta para ser cumprida na íntegra e sem valorizar os saberes de cada um sobre a doença e suas formas de enfrentá-la. Entretanto é necessário acreditar no potencial de transformação dos indivíduos, o que demanda do profissional uma escuta qualificada centrada nas necessidades dos participantes para que, em conjunto e em compartilhamento de saberes e experiências, seja possível identificar possibilidades de enfrentamento ao alcance indivíduo. ${ }^{20}$

No que se refere à participação da equipe na gestão do trabalho, percebe-se que os profissionais participam das reuniões de equipe e que um deles faz parte do grupo que coordena o projeto da Secretaria Municipal de Saúde (SMS) denominado 'Saúde Integrada Formando Cidadãos':

[...] sim, há reuniões mensais $[\ldots](P 1)$.

[...] A maior parte das orientações para os usuários partem desse projeto. Aqui na unidade, toda a equipe tem um perfil de trabalho parecido, todo mundo participa, pega junto para que a gente possa dar um resultado positivo no individual $e$ no coletivo $[. .$.$] (P3).$

Todos os profissionais participantes do estudo revelam participar de atividades de educação permanente, o que confirma a participação da equipe nos momentos coletivos. Para a PNH, a gestão participativa é valiosa na construção da mudança, contribuindo para um atendimento mais eficaz/efetivo e motivador para as equipes de trabalho. ${ }^{18}$ É positiva a participação de um profissional na coordenação do projeto da SMS, pois abre a possibilidade de inserção da realidade vivenciada na UBS.

\section{CONSIDERAÇÕES FINAIS}

Esse estudo promoveu um processo reflexivo na medida em que vislumbrava identificar a Política Nacional da Humanização nos cuidados com os usuários portadores de HAS/DM.

A limitação de registros sobre a história do usuário dificultou uma análise mais aprofundada do percurso do cuidado realizado pela equipe de saúde. Nesse sentido pontua-se a necessidade de registro no prontuário do paciente da assistência prestada por todos os profissionais. No que se refere aos enfermeiros, a consulta de enfermagem é obrigatória e regulamentada pela Resolução n³58/2009 do COFEN, mas não é prática cotidiana na assistência a portadores de HAS/DM.

A atuação do enfermeiro privilegia atividades em grupo em detrimento da consulta de enfermagem, 0 que reduz sua participação e contribuição no cuidado de portadores de HAS/DM, embora o papel do enfermeiro seja fundamental na promoção de programas e políticas de saúde.

No acolhimento, requisito da $\mathrm{PNH}$, identificou-se necessidade de organizar uma demanda programática para acesso dos portadores de HAS/DM aos serviços, bem como instituir um 


\section{JOURNALF \\ NURSING \\ AND HEALTH}

ISSN 2236 - 1987

acompanhamento individual periódico, conforme a necessidade individual.

Embora dispositivos disponíveis a partir da $\mathrm{PNH}$, o Projeto Terapêutico Singular e a Clínica ampliada não foram identificados na assistência. Foram identificadas iniciativas exitosas de gestão participativa e cogestão, tais como atividades de grupo e participação em projetos desenvolvidos no município. Há, entretanto, necessidade de avanços em relação à participação no Conselho Municipal de Saúde e à organização de conselhos locais de saúde, na perspectiva de promover a cidadania.

Conclui-se, que a implementação da PNH ainda se encontra em processo de apropriação (teórica e prática) no cuidado conduzido pela equipe saúde da família, a qual necessita de capacitações através da educação permanente, especialmente no que tange ao projeto terapêutico singular e à clínica ampliada.

\section{REFERÊNCIAS}

1. Malta DC, Silva MMA, Moura L, Morais NOL. A implantação do Sistema de Vigilância de Doenças Crônicas Não Transmissíveis no Brasil, 2003 a 2015: alcances e desafios. Rev bras epidemiol [Internet]. 2017 [acesso 2018 jul 09]; 20(4):661-75. Disponível em:

http: / /www.scielo.br/scielo.php?scrip $\mathrm{t}=\mathrm{sci}$ _arttext\&pid=S1415-

790X2017000400661\&lng=pt.

2. Malta DC, França E, Abreu DMX, Perillo RD, Salmen MC, Teixeira RA et al. Mortality due to noncommunicable diseases in Brazil, 1990 to 2015, according to estimates from the Global
Burden of Disease study. Sao Paulo Med J [Internet]. 2017 [cited 2018 Jul 09]; 135(3):213-21. Available from: http://www.scielo.br/scielo.php?scrip $\mathrm{t}=\mathrm{sci}$ arttext\&pid=S1516-

$31802017000300213 \&$ lng=en.

3. Oliveira VS, Maia PCGGS, Santo J, Sousa MNA. Análise dos fatores de risco para doenças crônicas não transmissíveis: estudo com colaboradores de uma instituição privada. Saude (Santa Maria) [Internet]. 2017 [citado 2018 jul 09]; 43(1):214-24. Disponível em: https: / / periodicos.ufsm.br/revistasau de/article/download/23784/pdf

4. Mafra LR, Birolo IVB, Tomasi CD, Ceretta LB, Soratto J, Losso ARS. Prática assistencial de enfermagem para portadores de doenças crônicas não transmissíveis e uma Estratégia de Saúde da Família de Criciúma - SC: um relato de experiência. Revista inova saúde. 2017; 6(2).

5. Ministério da Saúde (BR). Núcleo Técnico da Política Nacional de Humanização. Política Nacional de Humanização: a humanização como eixo norteador das práticas de atenção e gestão em todas as instâncias do SUS. Brasília; 2004.

6. Ministério da Saúde (BR). DATASUS. Sistema de informação de atenção básica 2014. Situação de saúde Rio Grande do Sul [Internet]. [acesso em 2014 nov 03]. Disponível em: http: / / tabnet.datasus.gov.br/cgi/tab cgi.exe?siab/cnv/SIABSrs

7. Minayo MC. $O$ desafio do conhecimento: pesquisa qualitativa em saúde. $14^{a}$ ed. São Paulo: Hucitec; 2014. 
ISSN 2236 - 1987

8. Tannure MC, Pinheiro AM. SAE: sistematização de assistência de enfermagem: guia prático. $2^{\text {a }}$ ed.[reimpressão] Rio de Janeiro: Guanabara Koogan; 2017.

9. Conselho Federal de Enfermagem (COFEN). Resolução COFEN-358/2009 [Internet]. [acesso em 2014 set 01]. Disponível em: http://www.cofen.gov.br/resoluocofen-3582009_4384.html

10. Malta DC, Campos MO, Oliveira MM, Iser BPM, Bernal RTI, Claro RM et al. Prevalência de fatores de risco e proteção para doenças crônicas não transmissíveis em adultos residentes em capitais brasileiras, 2013. Epidemiol serv saude [Internet]. 2015 [acesso 2018 jul 09]; 24(3):373-87. Disponível em: http://www.scielo.br/scielo.php?scrip $\mathrm{t}=\mathrm{sci}$ arttext\&pid=S2237-

$96222015000300373 \& \operatorname{lng}=e n$.

11. Garuzi M, Achitti COM, Sato CA, Rocha AS, Spagnuolo RS. Acolhimento na Estratégia Saúde da Família: revisão integrativa. Rev panam salud publica [Internet]. 2014 [acesso 2018 jul 09]; 2014;35(2)144-9. Disponível em: https://scielosp.org/pdf/rpsp/v35n2/ a09v35n2.pdf

12. Ministério da Saúde (BR). Secretaria de Atenção à Saúde. Departamento de Atenção Básica. Estratégias para o cuidado da pessoa com doença crônica. Brasília; 2014.

13 Collar JM, Neto JBA, Ferla AA. Formulação e impacto do Programa Mais Médicos na atenção e cuidado em saúde: contribuições iniciais e análise comparativa. Saúde em Redes [Internet]. 2015 [acesso em $2018 \mathrm{Jul}$ 02]; 1(2):43-56. Disponível em: http://revista.redeunida.org.br/ojs/i ndex.php/redeunida/article/view/591

14. Ministério da Saúde (BR). Secretaria de Atenção à Saúde. Política Nacional de Humanização. Atenção Básica. Brasília; 2010.

15. Coriolano-Marinus MWL, Queiroga BAM, Ruiz-Moreno L, Lima LS. Comunicação nas práticas em saúde: revisão integrativa da literatura. Saude soc [Internet]. 2014 [acesso 2018 jul 09]; 23(4):1356-69. Disponível em: http://www.scielo.br/scielo.php?scrip $\mathrm{t}=\mathrm{sci}$ arttext\&pid=S0104-

$12902014000401356 \&$ tng $=$ pt.

16. Boff L. Saber cuidar: ética do humano, compaixão pela terra. $9^{a}$ ed. Petrópolis: Vozes; 2014.

17. Silva RM, Peres ACO, Carcereri DL. A visita domiciliar como prática pedagógica na formação em odontologia. Ver ABENO [Internet]. 2017 [acesso 2018 jul 09]; 17(4):87-98. Disponível em: https://revabeno.emnuvens.com.br/r evabeno/article/view/515/344

18. Silva KS, Santos AM, Carvalho JÁ, Kochergin CN, Almeida PF. Percepção de gestores e enfermeiros sobre a organização do fluxo assistencial na rede de serviços de saúde. RECIIS (online) [Internet]. 2017 [acesso 2018 jul 09]; 11(2):1-12. Disponível em: https://www.reciis.icict. fiocruz.br/in dex.php/reciis/article/download/122 6/1226

19. Ministério da Saúde (BR). Política Nacional de Humanização da Atenção e da Gestão do SUS. Brasília; 2006.

20. Munari DB, Lucchese R, Medeiros M. Reflexões sobre o uso de atividades 


\section{JOURNALOF \\ NURSING \\ AND HEALTH}

grupais na atenção a portadores de doenças crônicas. Cienc cuid saude [Internet]. 2009 [acesso em $2018 \mathrm{Jul}$ 02];8(supl.):148-54. Disponível em: http://periodicos.uem.br/ojs/index.p hp/CiencCuidSaude/article/view/9742

Data de submissão: 31/10/2017

Data de aceite: $27 / 06 / 2018$

Data de publicação: 14/07/2018 